\title{
Some Observations on the Submicroscopic Structure of Cytoplasmic Particles Isolated from a Higher Plant
}

\author{
David P. Hackett
}

Department of Biology, University of Buffalo, New York, U.S.A.

Received November 16, $195 \%$

Although it is generally assumed that a common pattern of mitochondrial structure is found in all plant and animal cells (see Palade 1953; Sjöstrand 1956), the evidence for higher plants is very limited. It has been shown that plant mitochondria possess an external membrane, but their internal structure has not been widely studied (see Hackett 1955). Recent investigations (Farrant, Potter, Robertson and Wilkins 1956; Hodge, Martin and Morton 1957; Sager and Palade 1957) have demonstrated the presence of internal membranes, but the details of these structures have not been worked out, and there are some indications that they may differ somewhat from those found in most animal mitochondria. Even among various types of animal cells, there may be considerable variation in mitochondrial structure. The present paper reports on some electron microscopic observations of isolated cytoplasmic particles which may throw some light on the submicroscopic organization of plant mitochondria.

\section{Materials and methods}

Skunk-cabbage (Symplocarpvs foetidus L.) spadices were collected before the stamens had emerged from between the sepals. Flowers were cut off from the spadix and then ground in a glass mortar with sand in a medium containing $0.5 \mathrm{M}$ phosphate buffer ( $\mathrm{pH} 7.0$ ), and $0.001 \mathrm{M} \mathrm{MgSO}_{4}$. This and all subsequent operations were carried out at roughly $0^{\circ} \mathrm{C}$. After filtering through cheesecloth, the homogenate was centrifuged at $1,200 \times g$ (gravity) for five minutes, in order to sediment tissue fragments and large particles. The supernatant was then spun at $10,000 \times g$ for 30 minutes; the residue was washed by resuspending in isolating medium, with the aid of a glass homogenizer, and recentrifuging at $10,000 \times g$ for one hour to give the washed pellet.

With a glass rod, a small portion of the washed pellet was transferred to a test tube containing an appropriate fixing solution. In those cases where structural alterations were to be avoided, the solution contained $0.2 \mathrm{ml}$ of isolating medium and $0.1 \mathrm{ml}$ of a saturated $\mathrm{OsO}_{4}$ solution; final concentrations were $0.33 \mathrm{M}$ sucrose, $0.33 \mathrm{M}$ phosphate buffer, and roughly $2 \% \mathrm{OsO}_{4}$. 
Since the particles are sufficiently thick to scatter the incident electron beam almost completely, the examination of unaltered particles would reveal little structure. In order to expose structural details, it should be possible to disrupt the particles by exposing them to a solution of low tonicity, a treatment known to cause swelling or bursting. To accomplish this, isolated particles were added to $0.2 \mathrm{ml}$ of distilled water for a brief period (about five minutes), after which $0.1 \mathrm{ml}$ of $\mathrm{OsO}_{4}$ solution was added to fix the particles. All tubes were kept over-night in the refrigerator to allow adequate time for fixation.

Before preparing the mounts for electron microscopy, solutions containing sucrose were centrifuged and the particles washed with water. The fixed particles were resuspended and diluted with distilled water. A drop of this suspension was then placed on a Formvar film held by a copper screen. The excess water was withdrawn with a fine pipette and the preparation allowed to dry. The mounts were placed in vacuo for about four hours and then shadowed with chromium at an angle of 30 degrees; they were examined in an R.C.A. EMU $2 \mathrm{~B}$ electron microscope.

\section{Results}

The cytoplasmic components were first observed in situ by examining thin hand-sections of sepal tissue, which makes up most of the young skunkcabbage flower, in the phase microscope. The cells are packed with dense, spherical particles, roughly $1 \mu$ in diameter. In order to compare these granules with those isolated by centrifugation, a portion of the $10,000 \times \mathrm{g}$ pellet was also examined in the phase microscope. This appeared to be composed of a relatively homogeneous population of particles which resembled those seen in situ, both with respect to size and shape. Isolated particles that had been fixed with osmium in a sucrose-containing medium appeared as dense spheres in the electron microscope. In this case, the preparations showed considerable heterogeneity, with the particles ranging in diameter from 0.3 to $1.5 \mu$. Both in the phase and in the electron microscopes very little fine structure could be seen in the whole particles.

Those particles which had been exposed briefly to water before fixing with $\mathrm{OsO}_{4}$ were definitely less dense, more irregular in shape, and flatter than those described above, presumably because of the loss of internal substances during the "osmotic shock." In most cases (Fig. 1), they show clearly the presence of a thin external membrane around the relatively empty particles. Large folds or wrinkles in the membrane can be seen. The membrane is especially clear at the edges of the particles, and in some cases it appeared to be completely separated from the internal mass.

With respect to the internal organization, many of the disrupted particles showed regions of both high and low density. In some cases, granules 50 to $100 \mathrm{~m} \mu$ in diameter were present, either singly or in aggregates (Fig. 1). 
Larger and more irregular dark regions or bodies, 200 to $400 \mathrm{~m} \mu$ across, were also seen. In several of the preparations, uniform filamentous structures, about $35 \mathrm{~m} \mu$ wide, were seen outside the particles (Fig. 1). The filaments appear to be intimately interwoven with each other, although individual strands can be seen at the edges of the cluster. Since these filaments

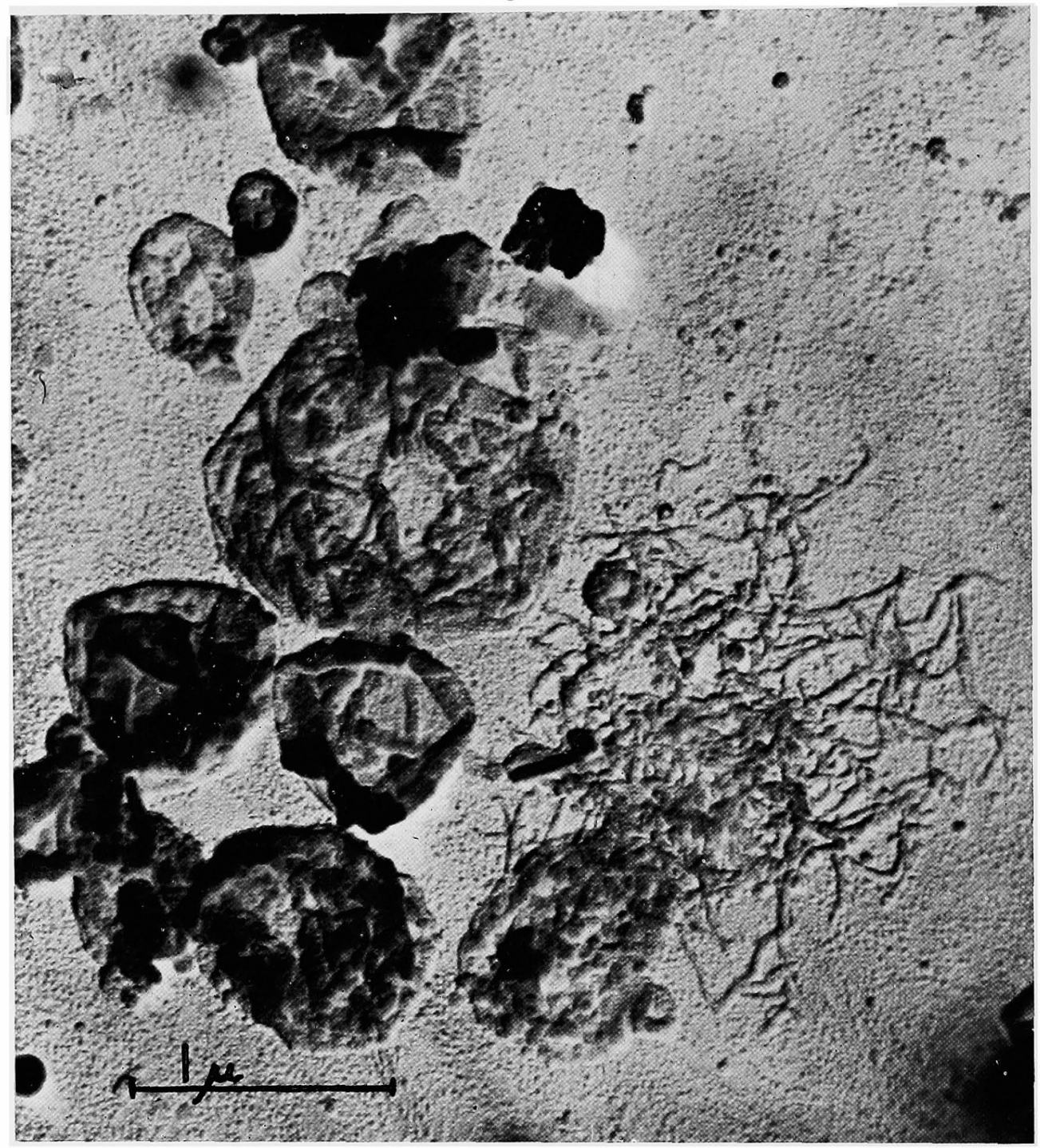

Fig. 1. Electron micrograph of cytoplasmic farticles isolated in sucrose-containing medium and exposed briefly to distilled water before fixation. Note external membrane, internal organization, and filaments both inside : nd outside particle.

were seen only when the particles had been exposed to water, the possibility that they originated from disintegrating particles must be considered. A careful examination of the largest granule in Fig. 1 supports this suggestion; in this case, it is clear that the filaments are present inside the particle, contained within the external membrane. The particle looks rather like a ball of yarn, with the filaments corresponding to individual strands. 


\section{Discussion}

The general morphology of the cytoplasmic particles observed in the intact cells suggests that they are mitochondria. The extraordinarily rapid $\mathrm{e}$ of respiration of this tisiue (Hackett 1957) may be related to the presence of large numbers of these particles, which can serve as respiratory centers. The isolated particles were sedimented by methods generally used for the preparation of mitochondria, and they possesss the characteristic biochemical properties, including the cytochrome system and the ability to oxidize Krebs cycle acids (Hackett 1957). Although other types of particles were probably present in the isolated preparations and conclusive identification of individual particles was not possible, the evidence suggests that those reported on here were mitochondria.

The method used in this study to reveal the fine structure of the isolated particles may be open to some objection. The technique of osmotic disruption can alter and distort the normal structural relations, and there are many difficulties inherent in the method of drop preparation. Even when the most suitable methods of embedding and thin-sectioning of the isolated pellet are employed (Farrant, Potter, Robertson and Wilkins 1956; Hodge, Martin and Morton 1957), some structural alterations result. Nevertheless, the preparations described here may provide some information on mitochondrial structure. The limiting membrane can be seen very clearly after osmotic disruption. The folds and wrinkles on the collapsed membranes are strikingly similar to those seen previously on isolated plant mitochondria (Farrant, Robertson and Wilkins 1953; Farrant, Potter, Robertson and Wilkins 1956).

The present study has confirmed the presence of considerable structure within the particles. In some cases, small spherical granules, 50 to $100 \mathrm{~m} \mu$ in diameter, were seen. Similar granules have been seen in isolated animal mitochondria only when there has been some disruption and loss of internal substances (Claude and Fullam 1945; Glimstedt, Lagerstedt and Ludwig, 1954), which suggests that they may be fragments of some other structure. The large, irregularly-shaped, osmiophilic body observed in some particles resembles the dense core or primary granum recently described in higher plant proplastids (Heitz 1954; Leyon 1954) and might correspond to a "mitochondrial corpuscle" (de Robertis and Reissig 1953). Of special interest is the nature of the filaments which were observed both inside and outside the particles. Although they might simply be some material (e.g. fibrous protein) precipitated during the preparation, the possibility that they represent major structural components can not be ignored. In the latter case, the particles might be composed of tightly woven and packed filaments, which could become unraveled on lysis. Such a system of coiled and interwoven elements would provide a large intramitochondrial surface area.

There have been several reports indicating that animal mitochondria may 
have a filamentous or tubular internal structure. In the male germ cells of Helix, the mitochondria apparently contain a number of filaments, $40 \mathrm{~m} \mu$ in diameter (Beams and Tahmisiam 1954). Studies on insects have shown that in the wing muscle of the bumble-bee (Philpott and Szent-Gyorgyi 1955) and in grasshopper Malpighian tubules (Beams, Tahmisian and Devine 1955) the mitochondria may contain a closely packed mass of tubules. Powers, Ehret and Roth (1955) have proposed that Paramecium mitochondria are made up of tightly packed tubules, each with a lumen $15 \mathrm{~m} \mu$ in diameter. This hypothesis has been supported recently by Wohlfarth-Botterman (1957) who depicts a Paramecium mitochondrion as a mass of "tubuli mitochondriales," each $29 \mathrm{~m} \mu$ in diameter. In the light of these reports, it seems possible that the filaments (roughly $35 \mathrm{~m} / \mu$ diameter) described here may represent tubular elements which normally make up some of the internal structure of the particles. Previous studies of the fine structure of plant mitochondria have shown that they contain internal membranous material (Palade 1953; Mercer, Hodge, Hope, and McLean 1955; Farrant, Potter, Robertson and Wilkins 1956; Sager and Palade 1957), and it has been concluded that they are true membranes (cristae) or lamellae, rather than filaments, even though they are "somewhat sinuous in appearance" (Hodge, Martin and Morton 1957). Nevertheless, the possibility that some higher plant mitochondria may possess a filamentous or tubular type of organization may be worth considering. Leyon (1954) has reported that the mitochondrialike proplastids of Aspidistra are made up of "what seems to be a net of anastomotic tubules in which a granular osmiophilic substance may be included."

\section{Summary}

The "mitochondrial fraction" isolated from young Skunk-cabbage (Symplocarpus foetidus) flowers was fixed with $\mathrm{OsO}_{4}$ and examined in the electron microscope. Particles which had been exposed briefly to water before fixation clearly showed an external membrane and an internal organization. Small granules, a larger dense body, and uniform filaments, approximately $35 \mathrm{~m} \mu$ wide, were seen within the particles. The possibility that these particles (mitochondria) are composed of an internal mass of interwoven filaments or tubules is considered.

\section{Acknowledgment}

The technical assistance of Mr. William Scharf and Mr. Harvey Sprowl is gratefully acknowledged. This work was aided in part by a grant from the National Science Foundation. 


\section{Literature cited}

Beams, H. W., and Tahmisian, T. N. 1954. Structure of the mitochondria in the male germ cells of Helix as revealed by the electron microscope. Exp. Cell Research 6: $87-93$.

-, - , and Devine, R. L. 1955. Electron microscope studies on the cells of the Malpighian tubules of the grasshopper (Orthoptera, Acrididae). J. Biophys. Biochem. Cytol. 1: $197-202$.

Claude, A., and Fullam, E. F, 1945. An electron microscope study of isolated mitochondria. J. Exp. Med, 81: 51-60.

DeRobertis, E., and Reissig, M. 1953. The submicroscopic organization of the ciliary and neuromotor apparatus of Tetrahymena Geleii. J. Appl. Physics 24: 1422.

Farrant, J. L., Potter, G., Robertson, R. N., and Wilkins, M. J. 1956. The morphology of red beet (Beta vulgaris L.) mitochondria. Austral. J. Bot. 4: 117-124.

-, Robertson, R. N. and Wilkins, M. J. 1953. The mitochondrial membrane. Nature 171: 401-402.

Glimstedt, G., Lagerstedt, S. and Ludwig, K. S. 1954. The visualization of the granulated mitochondrial inner body through treatment of isolated mitochondria with xylene. Experientia 10: $462-464$.

Hackett, D. P. 1955 . Recent studies on plant mitochondria. Int. Rev. Cyt. 4: 143-196.

- 1957. Respiratory mechanisms in the Aroid Spadix. J. Exp. Bot. 8: 157-171

Heitz, E. 1954. Kristallgitterstruktur des Granum junger Chloroplasten von Chlorophytum. Exp. Cell Research 7: 606-608.

Hodge, A. J., Martin, E. M., and Morton, R. K. 1957. The structure of some cytoplasmic components of plant cells in relation to the biochemical properties of isolated particles. J. Biophys. Biochem. Cyiol. 3; 61-70.

Leyon, H. 1954. The structure of chloroplasts IV. The development and structure of the Aspilistra chloroplast. Exp. Cell Research 7: 265-273.

Mercer, F. V., Hodge, A. J., Hope, A. B., and McLean, J. D., 1955. The structure and swelling properties of Nitella chloroplasts. Austral. J. Biol. Sci. 8: 1-18.

Palade, G. E. 1953. An electron microscope study of the mitochondrial structure. J Histochem. Cytochem. 1: 188-211.

Philpott, D. E., and Szent-Gyorgyi, A. 1955. Observations on the electron microscopic structure of insect muscle. Biochem. Biophys. Acta 18: 177-182.

Powers, E. L., Ehret, C. F., and Roth, L. E. 1955. Mitochondrial structure in Paramecium. as revealed by electron microscopy. Biol. Bull. 108: 182-195.

Sager, R., and Palade, G. E. 1957. Structure and development of the chloroplast in Chlamydomonas. I. The normal green cell. J. Biophys. Biochem. Cytol. 3: 463-488.

Sjöstrand, F. S. 1956. The ultrastructure of cells as revealed by the electron microscope. Int. Rev. Cytol. 5: 455-533.

Wohlfarth-Botterman, K. E. 1957. Die Enstehung, die Vermehrung und die Abscheidung geformter Sekrete der Mitochontrien von Paramecium. In Electron Microscopy, Proc. Stockholm Conference, 1956 (Ed. by F. S. Sjöstrand and J. Rhodin). Academic Press, New York. pp. 137-139. 